\title{
Compact sound-speed sensor for quartz enhanced photoacoustic spectroscopy based applications
}

\author{
Kun Liu, ${ }^{1,2, a)}$ Lei Dong, ${ }^{1,3}$ and Frank K. Tittel ${ }^{1, a)}$ \\ ${ }^{1}$ Department of Electrical and Computer Engineering, Rice University, 6100 Main St., Houston, \\ Texas 77005, USA \\ ${ }^{2}$ Anhui Institute of Optics and Fine Mechanics, Chinese Academy of Sciences, Hefei 230031, China \\ ${ }^{3}$ State Key Laboratory of Quantum Optics and Quantum Optics Devices, Institute of Laser Spectroscopy, \\ Shanxi University, Taiyuan 030006, China
}

(Received 11 January 2015; accepted 12 April 2015; published online 27 April 2015)

\begin{abstract}
A compact sound-speed sensor based on a phase difference method was developed. The sensor employs a U-shaped stainless steel tube with two holes located on its front and back ends, which serves as a sound wave guide. The phase difference between the two holes was measured using two mini-microphones by means of a phase-sensitive detection technique. This method offers the advantage of eliminating the influence of signal fluctuations. The frequency of a sound source offered by a loudspeaker can be scanned between $1 \mathrm{kHz}$ and $50 \mathrm{kHz}$. The slope of the phase difference as a function of frequency was obtained by scanning the frequency of the sound source. The speed of sound was retrieved from the rate of change of the phase difference. The performance of the sensor was evaluated over a wide range of speeds of sound from $260 \mathrm{~m} / \mathrm{s}$ to $1010 \mathrm{~m} / \mathrm{s}$ in different gas mixtures. The measured speed of sound was found to be in good agreement with the theoretical value for the sound-speed sensor. @ 2015 AIP Publishing LLC. [http://dx.doi.org/10.1063/1.4918796]
\end{abstract}

\section{INTRODUCTION}

Highly sensitive, selective, real-time, and compact trace gas sensors have a wide range of applications, such as environmental monitoring, climate research, agriculture, workplace safety, medical diagnostics, and industrial process control. ${ }^{1-3}$ There are many techniques that can be used to detect trace gases, such as integrated cavity output spectroscopy (ICOS), cavity ring down spectroscopy (CRDS), absorption spectroscopy with a multipass gas cell, and photoacoustic spectroscopy (PAS) ${ }^{4-7}$ Laser based optical absorption techniques offer the advantages of fast, real-time, and non-contact detection. Quartz enhanced photoacoustic spectroscopy (QEPAS) is a novel, rapidly developing photoacoustic spectroscopy technique for sensitive trace gas detection in a small sample volume by using a quartz tuning fork (QTF) (widely used in electronic watches and smartphones), as a detector of acoustic oscillations induced in an absorbing gas by modulated optical radiation. ${ }^{8}$ QEPAS has been successfully applied to the detection of numerous small molecules with narrow absorption spectra as well as several larger molecules with broad, unresolved spectral absorption features. ${ }^{9-17}$ Hence, QEPAS is a well-established technique to realize a highly sensitive, selective, real-time, and compact trace gas sensor. In most reported QEPAS based gas sensors, the spectrophone consists of a QTF and two pieces of rigid tubing placed along the excitation laser beam and positioned close to, but not touching the QTF, with two 30-50 $\mu \mathrm{m}$ gaps. Such a configuration serves as an absorption detection module (ADM). ${ }^{18}$ The two tubes form an acoustic micro-resonator, which concentrates

\footnotetext{
a) Authors to whom correspondence should be addressed. Electronic addresses: liukun@aiofm.ac.cn and fkt@ rice.edu
}

the acoustic pressure and, when properly designed, provide an additional resonant acoustic enhancement factor ranging from 10 to $30 .{ }^{18}$ Design parameters and enhancement of the micro-resonator are dependent on the properties of the target gas, in particular, the gas density and the speed of sound.

Most QEPAS sensors reported to date are designed for monitoring the concentration of one or several trace chemical species in a various carrier gases. Typical examples include the detection of $\mathrm{CH}_{4}, \mathrm{H}_{2} \mathrm{O}$ in air or $\mathrm{N}_{2},{ }^{13,14}$ or $\mathrm{NH}_{3}$ and $\mathrm{NO}$ in breath. ${ }^{19-21}$ In this case, the enhancement factor of the micro-resonator is a constant. However, there is a wide range of applications where the carrier gas composition can vary. Hence, the speed of sound in the carrier gas can change and influence the QEPAS signal, as the enhancement factor of the micro-resonator is not constant for different carrier gases. In this case, the speed of sound must be measured in real time to calibrate the QEPAS signal. For example, one application is the monitoring of $\mathrm{H}_{2} \mathrm{~S}$ concentration levels in the gas diffusing through the fluid isolation layer of flexible oil risers ${ }^{22}$ in which the carrier gas compositions can vary from $100 \% \mathrm{CO}_{2}$ to $100 \%$ $\mathrm{CH}_{4}$. In this case, the corresponding range of speeds of sound varies from $260 \mathrm{~m} / \mathrm{s}$ to $450 \mathrm{~m} / \mathrm{s}$. Such a range of speeds of sound will significantly influence the QEPAS signal, as shown in Fig. 1(a). The $\mathrm{CO}_{2}$ QEPAS signal does not increase linearly with the $\mathrm{CO}_{2}$ concentration. Fig. 1(b) shows the square of the $\mathrm{CO}_{2}$ QEPAS signal normalized to $\mathrm{CO}_{2}$ concentrations, which results in the resonance curve of the QEPAS micro-resonator as a function of the speed of sound. Hence, the exact speed of sound in the carrier gas must be measured to retrieve the $\mathrm{CO}_{2}$ concentration.

Several methods have been reported to measure the speed of sound, such as a pulse-echo technique. ${ }^{23-25}$ This technique retrieves the information of the speed of sound from the 

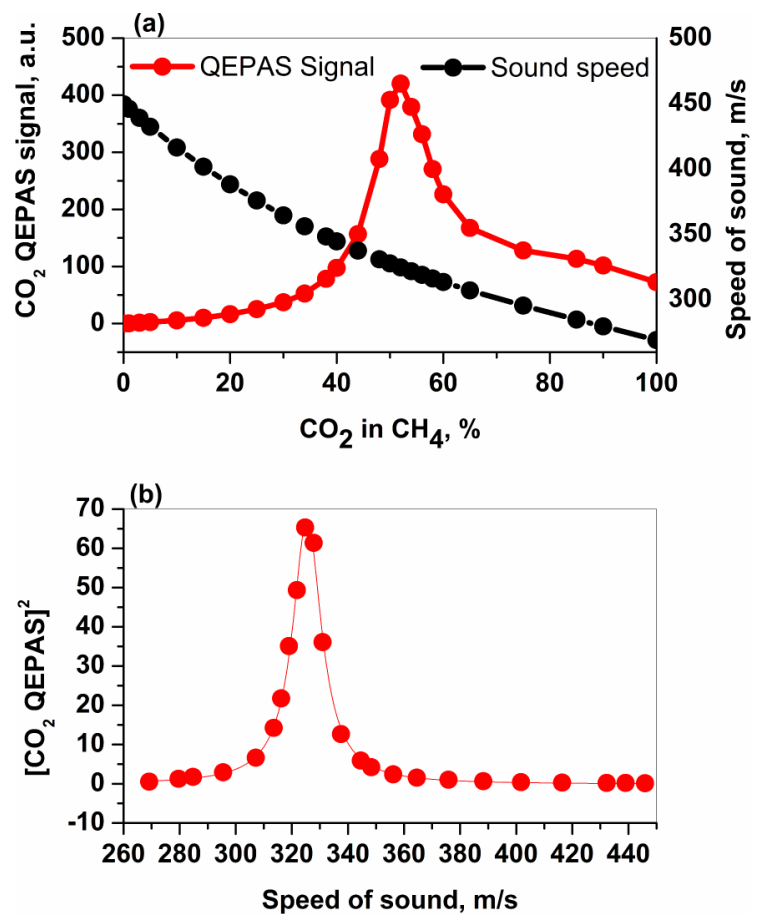

FIG. 1. (a) QEPAS signal as a function of $\mathrm{CO}_{2}$ concentration and corresponding speed of sound. (b) Square of QEPAS signal normalized to $\mathrm{CO}_{2}$ concentration and fitted by a Lorentzian curve.

distance between the transmitter and the receiver divided by the ultrasonic pulse travel time. This method requires a long distance between the transmitter and the receiver to obtain an acceptable accuracy. Therefore, such a sensor technique is difficult to realize in a compact size. However, a speed of sound sensor based on the phase shift comparison technique can be realized in a compact size as it measures the phase difference between two points. ${ }^{24}$ Theoretically, the distance between two points is as short as one wavelength for the phase difference method.

In this paper, we describe the development of a compact sound-speed sensor, which is based on a phase difference technique. The performance of the sound-speed sensor was evaluated in the range of speeds from $250 \mathrm{~m} / \mathrm{s}$ to $1000 \mathrm{~m} / \mathrm{s}$ in the carrier gas, and the results were found to be in good agreement with theoretical values.

\section{FUNDAMENTAL THEORY FOR SOUND SPEED MEASUREMENTS}

The speed of sound in a gas has been found to be sensitive to the properties of density of gases. The speed of sound is given by the following equation:

$$
c=\sqrt{\frac{\gamma p}{\rho}},
$$

where $\gamma$ is the specific heat ratio, $p$ is the pressure, and $\rho$ is the density. Using the ideal gas law with $p=n R T / V$ and $\rho=n M / V$, Eq. (1) becomes

$$
c=\sqrt{\frac{\gamma R T}{M}},
$$

where $R, T$, and $M$ are the universal gas constants, the absolute temperature (Kelvin), and molar mass, respectively.

When a sound wave with a frequency of $f$ propagates from location A to B separated by a distance $L$, the corresponding wave period $N$ is

$$
N=\frac{L f}{c} .
$$

As mentioned in the Sec. I, the conventional method for measuring the speed of sound is to measure the phase difference with a fixed distance $L$ and frequency $f$. In this case, the speed of sound $c$ is a function of the phase difference $\Delta \phi$ between the sent and received waves, ${ }^{24}$

$$
c=\frac{2 \pi f L}{\Delta \phi} .
$$

Generally, a phase comparator provides a linear voltage for a change in phase of up to $\pi$ radians. If the phase difference $\Delta \phi$ exceeds a value of $\pi$, non-valid measurements occur with this method. ${ }^{24}$

Furthermore, a determination of the phase difference with a fixed frequency $f$ and changing the distance $L$ was also used for measuring the speed of sound. In this case, the receiver (or transmitter) moves from original position $L_{0}$ to $L$. The phase difference $\Delta \phi$ is

$$
\Delta \phi=\left(L-L_{0}\right) \frac{2 \pi f}{c}=\Delta L \frac{2 \pi f}{c} .
$$

This method requires a motor to control, the locations of the receiver and transmitter, which complicates such a sensor system. $^{25}$

In this work, we employ a novel method that allows variation of the frequency $f$ with a fixed distance $L$, which is both convenient and accurate. Considering the case that the sound frequency varies from $f_{1}$ to $f_{2}$ in a fixed distance $L$, the corresponding wave periods are

$$
\begin{aligned}
& N_{1}=\frac{L f_{1}}{c}, \\
& N_{2}=\frac{L f_{2}}{c} .
\end{aligned}
$$

The phase difference is

$$
\Delta \phi=2 \pi\left(N_{2}-N_{1}\right)=\frac{2 \pi L}{c} \Delta f
$$

and

$$
\frac{d \phi}{d f}=\frac{2 \pi L}{c} .
$$

Therefore, by changing the frequency and recording the corresponding phase difference between point $\mathrm{A}$ and $\mathrm{B}$, the value of $d \phi / d f$ is obtained, which yields the speed of sound,

$$
c=2 \pi L /\left(\frac{d \phi}{d f}\right) .
$$

\section{SOUND-SPEED SENSOR DESIGN}

The sound-speed sensor architecture is shown in Fig. 2, which employs a phase shift method to measure the speed of 


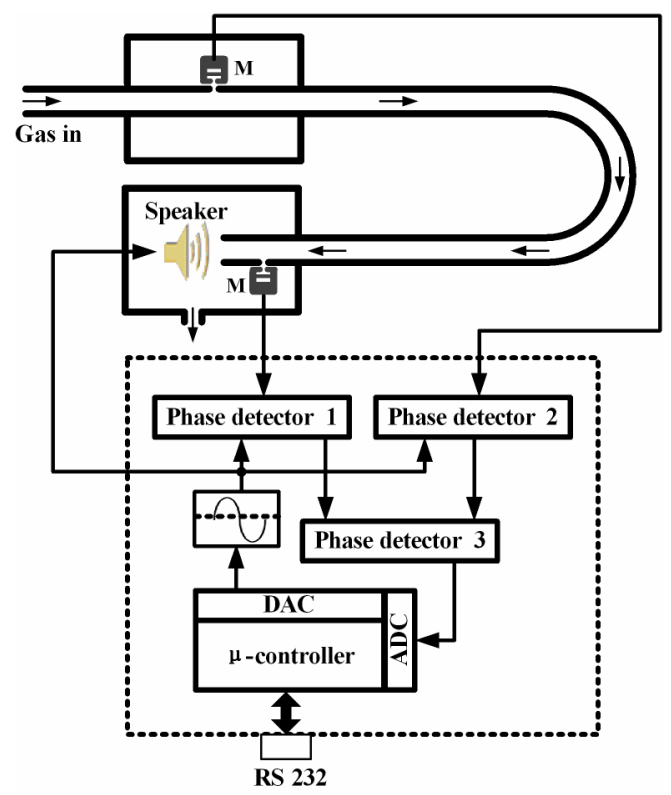

FIG. 2. Schematic of the sound speed sensor. M: mini-microphone.

sound. This technology consists of a microcontroller and a loudspeaker as well as two MEMS microphones (Knowles Acoustics SPM0404HE5H-PB) and a U-shaped stainless steel tube. The microcontroller consists of a digital signal processing (DSP)-controllable sine-wave generator and three phase detectors with a resolution of $0.0055^{\circ}$, which are mounted inside a compact box measuring $12 \times 8.5 \times 4 \mathrm{~cm}$. The microcontroller was designed to operate with a control electronics unit (CEU) developed in a previous QEPAS multicomponent gas sensor via $I^{2} \mathrm{C}$ communication. ${ }^{22}$ The $\mathrm{CEU}$ can be controlled with a personal computer. The frequency range of the sine wave is $1 \mathrm{kHz}-50 \mathrm{kHz}$ with a resolution of $0.01 \mathrm{~Hz}$ and can be scanned at a rate of $100 \mathrm{~ms} /$ point. The maximum number of scan points is 1024 . The range of amplitude of the sine wave is $0-5 \mathrm{~V}$ (peak to peak). Phase detector 1 receives the phase of the input signal from channel 1 relative to the sine wave from the generator. Phase detector 2 acquires the phase of the input signal from channel 2 relative to the sine wave from the generator. Phase detector 3 calculates the phase difference between channel 1 and channel 2 according to the phase value obtained by phase detectors 1 and 2 . Such a design offers the advantage of eliminating the interference of input signal fluctuations during measurements as there are always strong reference signals from the generator for phase detectors 1 and 2 . Thus, an accurate phase value can be obtained even with weak signals from channels 1 and 2 . A counter in the microcontroller measures the period of the phase difference. When the phase difference covers a range from $0^{\circ}$ to $360^{\circ}$, the counter decreases by 1 , and increases by 1 if the phase difference decreases from $360^{\circ}$ to $0^{\circ}$. This method allows a phase difference measurement that exceeds one period. Two miniature MEMS microphones with a $0.84 \mathrm{~mm}$ diameter acoustic hole are used to detect the phase of the sound wave.

A sine wave voltage from the microcontroller was applied to the loudspeaker to generate a sine sound wave. An $18 \mathrm{~cm}$ long U-shape stainless steel tube with an outer diameter of $2.1 \mathrm{~mm}$ and inner diameter of $1.6 \mathrm{~mm}$ was used as a sound guide. One end of the tube was placed in front of the loudspeaker, so that the sound generated by the loudspeaker was propagated in the tube. The tube has two small holes with a $1 \mathrm{~mm}$ diameter for detecting the phase difference between them. Hole 1 was located $1 \mathrm{~cm}$ from the front end of the tube, which is close to the loudspeaker. Microphone 1 was placed opposite to hole 1 in order to detect the sound signal at hole position 1 . Hole 2 was set close to the end facet of the tube. The length of the tube between two holes is $14.82 \mathrm{~cm}$. Microphone 2 was placed opposite to hole 2 to detect the sound signal at the position of hole 2. A novel compact sensor configuration is obtained by employing the U-shaped tube instead of using a straight tube. The amplitude of the input signal was $0.125 \mathrm{~V}$ or $1.25 \mathrm{~V}$ (peak to peak) and depends on the gain factor. Two red and two green light-emitting diodes (LEDs) on the sensor enclosure indicate whether the output data are acceptable or not. The red LEDs indicate overload conditions of the corresponding channel when the output data are inaccurate. The green LEDs indicate whether the input signals of the corresponding channels are within a range that guarantees processing precision. When one of the green LEDs is off, the input signal of the corresponding channel is $<10 \%$ of the full range, and the processing accuracy is decreased. Therefore, when a red LED is on or a green LED is off, it is necessary to decrease or increase the sine wave amplitude, respectively. A prototype of the developed sound-speed sensor is shown in Fig. 3.

When measuring the speed of sound, the microcontroller scans the frequency of the sine wave and records the difference of phase obtained by phase detector 3 at each frequency. This measurement provides the slope of the phase as a function of the frequency by a linear fit routine, after completing a scan. With such a technique, errors caused by sound reflection or resonance in the tube are reduced. Therefore, the speed of sound can be calculated from the results of the slope by using Eq. (9). All measurements and parameter settings including start and end of scanning frequency, frequency period of scanning, and completion of sine wave amplitude as well as the gain of the input signal were performed using a LabView software based program.

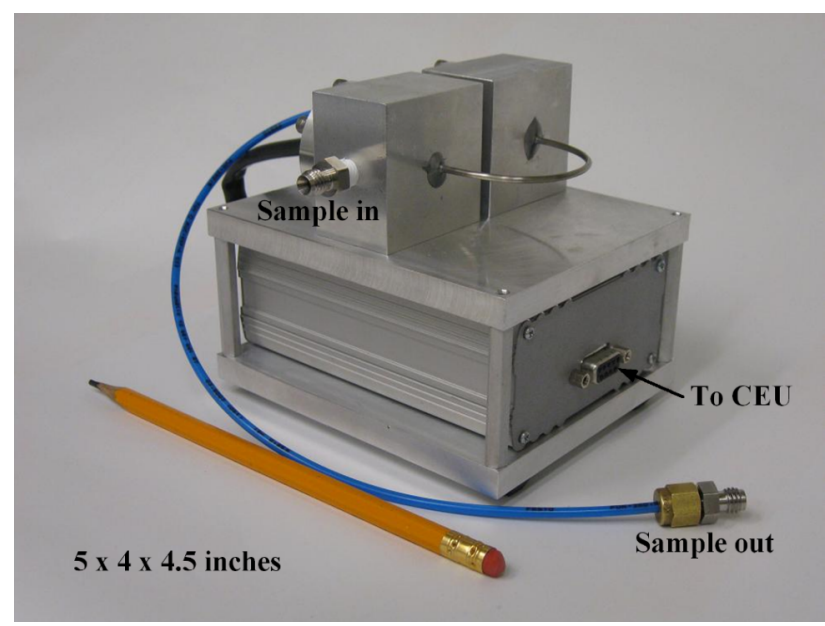

FIG. 3. Photograph of the sound-speed sensor. 


\section{SENSOR PERFORMANCE EVALUATION}

Compared to the sound speed sensor based on a Ushaped stainless tube, the performance of the sound speed sensor based on a straight stainless steel tube design was first evaluated. A high frequency ultrasonic wave was used and the main errors are from the sound reflection and resonance in the tube when the phase shift method is used. An $18 \mathrm{~cm}$ long straight stainless steel tube with the same length as the U-shaped stainless tube was used as a sound wave guide. The sufficient length eliminates the reflection and the resonance of sound in the tube. The tube has two holes, hole 1 and hole 2 , for measuring the phase of the sound wave, respectively. The distance between hole 1 and the front end of the tube was $1 \mathrm{~cm}$. The distance between hole 1 and hole 2 was $\sim 15 \mathrm{~cm}$. The exact distance between holes 1 and 2 was calibrated in pure nitrogen by means of the calculated sound speed and Eq. (8). Nitrogen flowed into the tube at normal atmospheric pressure and at a temperature of $22^{\circ} \mathrm{C}$. The flow rate was decreased to $0 \mathrm{sccm}$ after filling the tube with pure nitrogen. Subsequently, the frequency of the sine wave was scanned from $20 \mathrm{kHz}$ to $40 \mathrm{kHz}$ in $100 \mathrm{~Hz}$ steps to obtain the phase slope as a function of frequency. Fig. 4 shows the measured phase difference as a function of frequency with a measured slope of 0.153. Using Eq. (2) and the parameters of pure nitrogen, including the ratio of the specific heat $\gamma=1.4$ and the molar mass $\boldsymbol{M}=28.02 \mathrm{~g} / \mathrm{mol}$, the calculated speed of sound is $349.98 \mathrm{~m} / \mathrm{s}$ in pure nitrogen at $22^{\circ} \mathrm{C}$. From Eq. (8), we obtain a distance, $L$,

$$
L=c \frac{d \phi}{d f} \mid(2 \pi) .
$$

Therefore, from the slope parameters, the speed of sound in pure nitrogen, and using Eq. (10), the exact distance between holes 1 and 2 is determined to be $14.82 \mathrm{~cm}$.

As described in Sec. III, the reported sound speed sensor was designed for a QEPAS gas sensor. Generally, a QEPAS based gas sensor is operated with a gas flow rate from $0 \mathrm{sccm}$ to $200 \mathrm{sccm}$. Therefore, a measurement with a flow rate of $200 \mathrm{sccm}$ in the tube was carried out in order to investigate the influence of the gas flow rate on the result of measured

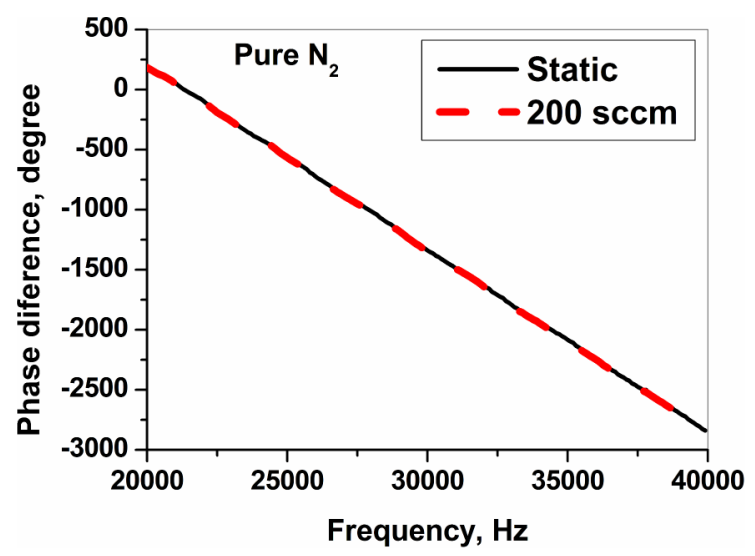

FIG. 4. Phase difference as a function of frequency of sound in pure nitrogen. Black line, data measured in static pure nitrogen; red line, data measured in nitrogen with a flow rate of $200 \mathrm{sccm}$.

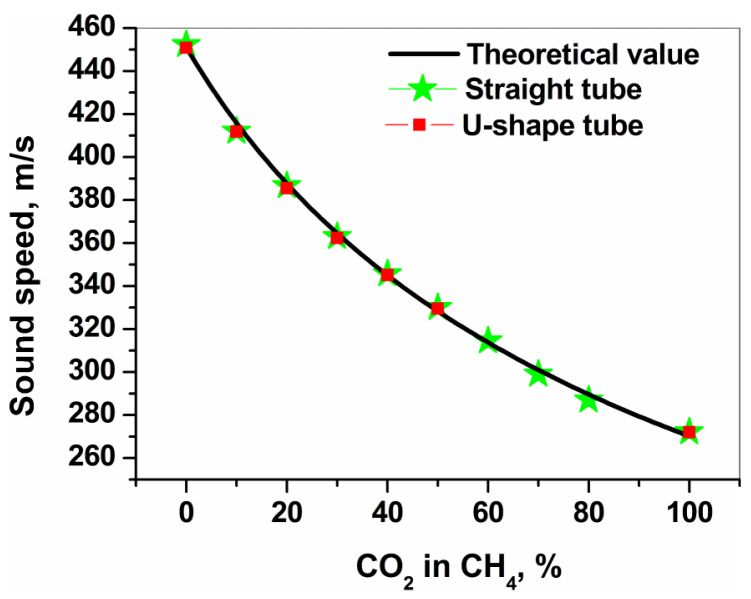

FIG. 5. Speed of sound in a carbon dioxide $\left(\mathrm{CO}_{2}\right)$-methane $\left(\mathrm{CH}_{4}\right)$ mixture measured by using a straight tube and U-shaped tube, respectively.

sound speed. Figure 4 depicts the measured difference of phase as a function of frequency in both cases of $200 \mathrm{sccm}$ and no flow. The measured sound speed was $349.43 \mathrm{~m} / \mathrm{s}$ and $349.98 \mathrm{~m} / \mathrm{s}$, respectively. This illustrates that the influence of a $200 \mathrm{sccm}$ flow rate on the speed of sound and can in fact be neglected. Fig. 5 shows that the measured speeds of sound in $\mathrm{CO}_{2}$ and $\mathrm{CH}_{4}$ mixtures are in good agreement with the expected theoretical values.

The value of $d \phi / d f$ is proportional to the distance $\boldsymbol{L}$ as described by Eq. (8). Therefore, a straight tube with a longer distance will offer a more accurate value for the measurement of speed of sound. However, a longer tube will increase the sensor size. Hence, the straight stainless steel tube used in above reported measurements was changed to a U-shaped tube configuration to decrease the size of the sound speed sensor. A series of measurements were carried out to evaluate the performance of such a modified sound-speed sensor. The speed of sound in $\mathrm{CO}_{2}$ and $\mathrm{CH}_{4}$ gas mixtures was measured with a flow rate of $\sim 200 \mathrm{sccm}$ and compared with the previous results obtained for a straight tube sensor configuration, as shown in Fig. 5. The two results shown in Fig. 5 are in good agreement. This proves that a U-shaped stainless steel tube operates as well as a straight stainless steel tube. There are no additional reflection and resonance

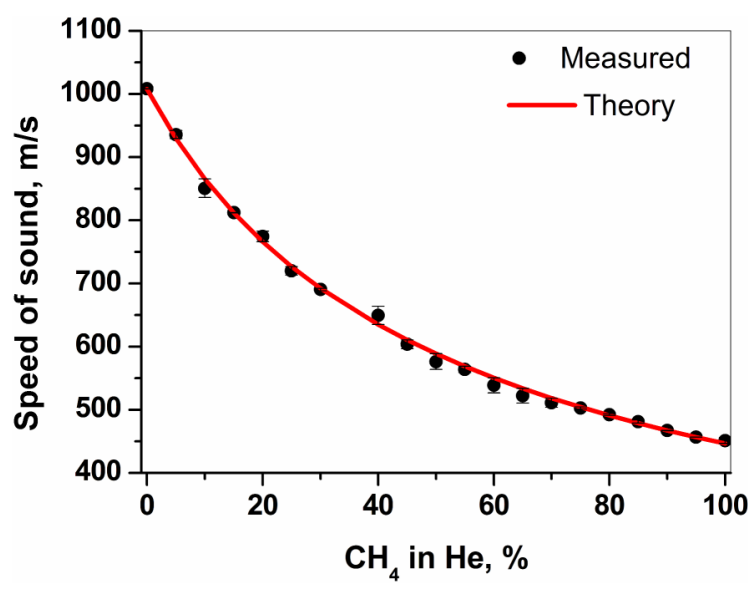

FIG. 6. Speed of sound in a methane $\left(\mathrm{CH}_{4}\right)$-helium $(\mathrm{He})$ mixture. 


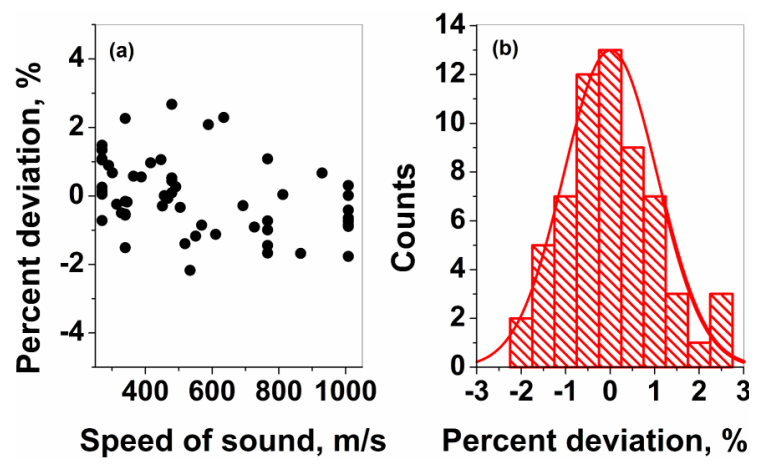

FIG. 7. (a) Percent deviation of the measured sound speed compared with the calculated value. (b) Histogram plot of the deviation. The red line depicts a Gaussian profile.

issues in a U-shaped stainless steel tube. Such a U-shaped tube significantly decreases the sensor size.

For testing the performance of the speed of sound sensor for different gas mixtures, the speed of sound was measured in mixtures of $\mathrm{CH}_{4}$ and $\mathrm{CO}_{2}$, mixtures of $\mathrm{CH}_{4}$ and $\mathrm{He}$, as well as pure $\mathrm{CH}_{4}, \mathrm{CO}_{2}$, and $\mathrm{He}$, respectively. The corresponding sound speed ranges from $260 \mathrm{~m} / \mathrm{s}$ (pure $\mathrm{CO}_{2}$ ) to $450 \mathrm{~m} / \mathrm{s}$ (pure $\mathrm{CH}_{4}$ ) and from $450 \mathrm{~m} / \mathrm{s}$ to $1010 \mathrm{~m} / \mathrm{s}$ (pure He), respectively. The gas mixtures were obtained by using a commercial gas dilution system (Series 4040, Environics, Inc.). The scanning range of the sine wave was from $25 \mathrm{kHz}$ to $40 \mathrm{kHz}$ in steps of $100 \mathrm{~Hz}$ during such measurements. Figs. 5 and 6 show the results measured with different mixtures, and the black lines in both figures depict the calculated values. The theoretical values of the sound speed are calculated using Eq. (1). In this calculation, the parameters of $\mathrm{M}, c_{p}$, and $c_{v}$ are calculated as weighted mean values according to the volume gas fractions in the mixture. The heat capacity at constant pressure is $c_{p}=31.136 \mathrm{~J} /(\mathrm{Mol} \mathrm{K})$ and $c_{v}=28.82 \mathrm{~J} /(\mathrm{Mol} \mathrm{K})$ for $\mathrm{CO}_{2}$, $c_{p}=35.52 \mathrm{~J} /(\mathrm{Mol} \mathrm{K})$ and $c_{v}=27.2 \mathrm{~J} /(\mathrm{mol} \mathrm{K})$ for $\mathrm{CH}_{4}$, and $c_{p}=20.76 \mathrm{~J} /(\mathrm{mol} \mathrm{K})$ and $c_{v} 12.48 \mathrm{~J} /(\mathrm{mol} \mathrm{K})$ for He.$^{26}$ Hence, the measured speeds of sound are in good agreement with theoretical values.

Fig. 7(a) shows that the percent deviations between the measured and the calculated values, which were obtained by first subtracting the measured value from the calculated value and then by dividing the calculated value and multiplying by 100. The maximum errors of the sensor are $<2.3 \%$ in the entire range of sound speeds from $260 \mathrm{~m} / \mathrm{s}$ to $1010 \mathrm{~m} / \mathrm{s}$ compared to the theoretical value. The standard deviation of the measured errors was found to be $<1 \%$. Fig. 7(b) depicts the histogram plot of the deviation data in the Fig. 7(a), which was found to be a Gaussian distribution. The half width at half maximum (HWHM) was found to be $1.01 \%$ of the Gaussian profile. This verifies that the sensor measurement precision is better than $1 \%$. Hence, our results illustrate that the reported sound-speed sensor offers high precision measurement results.

\section{CONCLUSIONS}

A compact sound-speed sensor based on a phase difference method was developed. The sensor employs a U-shaped stainless steel tube with two holes. Such a design was experimentally shown to perform equally well as a straight sensor tube. In addition, the design offers sufficient length between two holes with a compact size to eliminate or minimize the influence of reflection and the sound resonance in the tube. The performance of the sensor was evaluated over a wide range of speeds of sound from $260 \mathrm{~m} / \mathrm{s}$ to $1010 \mathrm{~m} / \mathrm{s}$ in a mixture of different gases, and the results are in good agreement with the theoretical values with an error of $<1 \%$. Errors may originate from the resonance or the reflection of sound in the sensor tube which in turn affect the sound phase. ${ }^{24}$ In this work, the error may also arise from a fractional error of each component in the mixture, which can cause an error of the theoretical value. To improve the accuracy of the sensor, we can increase the range of scanning of the sine wave or replace the U-shaped stainless steel tube with a longer length tube as a sound wave guide. The reported sound speed sensor was designed for QEPAS applications of multi-component trace gas sensors in order to correct the QEPAS signals. Furthermore, the sensor can also be used in applications that require the measurement of the speed of sound in gases. For example, such a sound-speed sensor can be used to measure gas temperatures ${ }^{25}$ analyze the quality of gases, and measure the compositions of binary gas mixtures. ${ }^{27-29}$

\section{ACKNOWLEDGMENTS}

The authors gratefully acknowledge the financial support from a National Science Foundation (NSF) ERC MIRTHE award, a NNSF-ANR award NexCILAS, a Grant No. C0586 from the Robert Welch Foundation and National Natural Science Foundation of China (Nos. 41205120, 41175036, 61275213, and 61108030).

${ }^{1}$ G. Wysocki, Y. Bakhirkin, S. So, F. K. Tittel, C. J. Hill, R. Q. Yang, and M. P. Fraser, "Dual interband cascade laser based trace-gas sensor for environmental monitoring," Appl. Opt. 46, 8202 (2007).

${ }^{2}$ T. H. Risby and F. K. Tittel, "Current status of mid-infrared quantum and interband cascade lasers for clinical breath analysis," Opt. Eng. 49, 111123 (2010).

${ }^{3}$ I. Linnerud, P. Kaspersen, and T. Jager, "Gas monitoring in the process industry using diode laser spectroscopy," Appl. Phys. B 67, 297 (1998).

${ }^{4}$ G. Berden, R. Peeters, and G. Meijer, "Cavity ring-down spectroscopy: Experimental schemes and applications," Int. Rev. Phys. Chem. 19, 565 (2000).

${ }^{5}$ J. B. McManus, P. L. Kebabian, and M. S. Zahniser, "Astigmatic mirror multipass absorption cells for long-path-length spectroscopy," Appl. Opt. 34, 3336 (1995).

${ }^{6}$ W. Chen, A. A. Kosterev, and F. K. Tittel, " $\mathrm{H}_{2} \mathrm{~S}$ trace detection using offaxis integrated cavity output spectroscopy in the near-infrared," Appl. Phys. B 90, 311 (2008).

${ }^{7}$ M. W. Sigrist and C. Fischer, "Perspectives of laser-photoacoustic spectroscopy in trace gas sensing," J. Phys. IV 125, 619 (2005).

${ }^{8}$ A. A. Kosterev, Y. A. Bakhirkin, R. F. Curl, and F. K. Tittel, "Quartzenhanced photoacoustic spectroscopy," Opt. Lett. 27, 1902 (2002).

${ }^{9}$ A. A. Kosterev and F. K. Tittel, "Ammonia detection by use of quartzenhanced photoacoustic spectroscopy with a near-IR telecommunication diode laser," Appl. Opt. 43, 6213 (2004).

${ }^{10}$ R. Lewicki, G. Wysocki, A. A. Kosterev, and F. K. Tittel, "Carbon dioxide and ammonia detection using $2 \mu \mathrm{m}$ diode laser based quartz-enhanced photoacoustic spectroscopy," Appl. Phys. B 87, 157 (2007).

${ }^{11}$ A. A. Kosterev, T. S. Mosely, and F. K. Tittel, "Impact of humidity on quartzenhanced photoacoustic spectroscopy based detection of HCN," Appl. Phys. B 85, 295 (2006).

${ }^{12}$ S. Schilt, A. A. Kosterev, and F. K. Tittel, "Performance evaluation of a near infrared QEPAS based ethylene sensor," Appl. Phys. B 95, 813 (2009).

${ }^{13}$ A. A. Kosterev, Y. A. Bakhirkin, F. K. Tittel, S. Mcwhorter, and B. Ashcraft, "QEPAS methane sensor performance for humidified gases," Appl. Phys. B 92, 103 (2008). 
${ }^{14}$ K. Liu, H. Yi, A. A. Kosterev, W. Chen, L. Dong, L. Wang, T. Tan, W. Zhang, F. K. Tittel, and X. Gao, "Trace gas detection based on off-beam quartz enhanced photoacoustic spectroscopy: Optimization and performance evaluation,” Rev. Sci. Instrum. 81, 103103 (2010).

${ }^{15}$ M. Horstjann, Y. A. Bakhirkin, A. A. Kosterev, R. F. Curl, F. K. Tittel, C. M. Wong, C. J. Hill, and R. Q. Yang, "Formaldehyde sensor using interband cascade laser based quartz-enhanced photoacoustic spectroscopy," Appl. Phys. B 79, 799 (2004).

${ }^{16}$ M. C. Phillips, T. L. Myers, M. D. Wojcik, and B. D. Cannon, "External cavity quantum cascade laser for quartz tuning fork photoacoustic spectroscopy of broad absorption features," Opt. Lett. 32, 1177 (2006).

${ }^{17}$ R. Lewicki, G. Wysocki, A. A. Kosterev, and F. K. Tittel, "QEPAS based detection of broadband absorbing molecules using a widely tunable, $\mathrm{cw}$ quantum cascade laser at $8.4 \mu \mathrm{m}$," Opt. Express 15, 7357 (2007).

${ }^{18}$ L. Dong, A. A. Kosterev, D. Thomazy, and F. K. Tittel, "QEPAS spectrophones: Design, optimization, and performance," Appl. Phys. B 100, 627 (2010).

${ }^{19}$ R. Lewicki, A. A. Kosterev, D. M. Thomazy, T. H. Risby, S. Solga, T. B. Schwartz, and F. K. Tittel, "Real time ammonia detection in exhaled human breath using a distributed feedback quantum cascade laser based sensor," Proc. SPIE 7945, 79450K (2011).

${ }^{20}$ L. Dong, V. Spagnolo, R. Lewicki, and F. K. Tittel, "Ppb-level detection of nitric oxide using an external cavity quantum cascade laser based QEPAS sensor," Opt. Express 19, 24037-24045 (2011).
${ }^{21}$ V. Spagnolo, A. A. Kosterev, L. Dong, R. Lewicki, and F. K. Tittel, "NO trace gas sensor based on quartz-enhanced photoacoustic spectroscopy and external cavity quantum cascade laser," Appl. Phys. B 100, 125-130 (2010).

${ }^{22}$ A. A. Kosterev, L. Dong, D. Thomazy, F. K. Tittel, and S. Overby, "QEPAS for chemical analysis of multi-component gas mixtures," Appl. Phys. B 101, 649 (2010).

${ }^{23}$ J. F. Figueroa and J. S. Lamancusa, "A method for accurate detection of time of arrival: Analysis and design of an ultrasonic ranging system," J. Acoust. Soc. Am. 91, 486 (1992).

${ }^{24}$ J. S. Olfert, M. D. Checkel, and C. R. Koch, "Acoustic method for measuring the sound speed of gases over small path lengths," Rev. Sci. Instrum. 78, 054901 (2007).

${ }^{25}$ K. N. Huang, C. F. Huang, Y. C. Li, and M. S. Young, "High precision, fast ultrasonic thermometer based on measurement of the speed of sound in air," Rev. Sci. Instrum. 73, 4022 (2002).

${ }^{26}$ See www.engineeringtoolbox.com for information about heat capacity of gases.

${ }^{27}$ L. Ruffine and J. P. M. Trusler, "Sound-speed sensor for gas pipeline applications," Int. J. Thermophys. 30, 1106-1117 (2009).

${ }^{28}$ E. Polturak, S. L. Garret, and S. G. Lipson, "Precision acoustic gas analyzer for binary mixtures," Rev. Sci. Instrum. 57, 2837 (1986).

${ }^{29}$ J. S. Brooks and R. B. Hallock, "Simple apparatus for concentration determinations in binary gas mixtures," Rev. Sci. Instrum. 54, 1199 (1983). 